\title{
Independent Resolving Number of Fibonacci Cubes and Extended Fibonacci Cubes
}

\author{
Chris Monica M. \\ Department of Mathematics \\ Loyola College \\ Chennai 600034
}

\author{
D. Little Femilin Jana \\ Department of Mathematics \\ Prince Shri Venkateshwara Padmavathy \\ Engineering College, Chennai 600127
}

\begin{abstract}
A subset $S$ of vertices in a graph $G$ is said to be an independent set of $G$ if each edge in the graph has at most one endpoint in $S$ and a set $W \subseteq V$ is said to be a resolving set of $G$, if the vertices in $G$ have distinct representations with respect to $W$. A resolving set $W$ is said to be an independent resolving set, or an $i r$-set, if it is both resolving and independent. The minimum cardinality of $W$ is called the independent resolving number and is denoted by $\operatorname{ir}(G)$. In this paper, we determine the independent resolving number of Fibonacci Cubes and Extended Fibonacci cubes.
\end{abstract}

\section{General Terms}

Graph theory, Interconnection network, Binary representation

\section{Keywords}

Resolving set, Independent resolving number, Fibonacci Cubes, Extended Fibonacci Cubes, Hamming distance.

\section{INTRODUCTION}

For an ordered set $W=\left\{w_{1}, w_{2}, \ldots, w_{k}\right\}$ subset of $V$ and a vertex. $v \in V(G)$, the representation of $v$ with respect to $W$ is defined as the $k$-vector $r(v \mid W)=\left(d\left(v, w_{1}\right), d\left(v, w_{2}\right), \ldots, d\left(v, w_{k}\right)\right)$. The set $W$ is a resolving set of $G$ if $r(x \mid W) \neq r(y \mid W)$ for any two distinct vertices $x, y \in V$. A minimum resolving set or a basis of $G$ is a resolving set of $G$ with minimum number of vertices. The metric (dimension) $\operatorname{dim}(G)$ is the number of vertices in a basis for $G$. A resolving set $W$ of $G$ is connected if the sub graph $\langle W\rangle$ induced by $W$ is a connected sub graph of $G$ and the minimum cardinality of a connected resolving set $W$ in a graph $G$ is called the connected resolving number which is denoted by $\operatorname{cr}(G)$ [1]. A set $W \subseteq V$ is said to be an independent set of $G$ if there is no edge connecting every two vertices in $W$. A resolving set $W$ is said to be an independent resolving set, or an ir-set, if it is both resolving and independent. The cardinality of a minimum independent resolving set in a graph $G$ is known as the independent resolving number $\operatorname{ir}(G)$. [1]

For every connected graph $G$ of order $n$, every independent resolving set is a resolving set. Saenpholphat et al. [1] have proved that $1 \leq \operatorname{dim}(G) \leq \operatorname{ir}(G) \leq \beta(G) \leq n-1$ where $\operatorname{dim}(G)$ is the number of vertices in a basis for $G$ and $\beta(G)$ is the number of vertices in a maximum independent set in a graph $G$.

One of the basic problems in chemistry is to provide distinct mathematical representations for a set of chemical compounds. The structure of a chemical compound is labeled graphically where the vertex and edge labels specify the atom and bond types, respectively. Under graph theoretical concept, this problem is to find a resolving set of the graph. Further this concept has wide applications in problems of network discovery and verification [2], pattern recognition and image processing, coin weighing problems, strategies for master mind game, geometrical routing protocols, sonar and loran stations [3], pharmaceutical chemistry, Combinatorial Search and Optimization, robot navigation, etc.

In this paper, we determine the independent resolving number of Fibonacci cube, Extended Fibonacci cubes $\operatorname{EFC}_{1}(n)$ and $\operatorname{EFC}_{2}(n)$

\section{FIBONACCI CUBES AND EXTENDED FIBONACCI CUBES}

One of the most popular and efficient topological structure of interconnection network is hypercube [4]. This led to the introduction of a special sub cube of hypercube, called Fibonacci cube proposed by Hsu [5]. Fibonacci cubes are the sub graphs of hypercube induced by the vertices that no two consecutive 1's are there in the binary representation. When comparing the hypercube of dimension $n$ with an n-dimensional Fibonacci cubes, it is found that there are $1 / 5$ fewer edges [6] and does not increase rapidly in size as the dimension increases. Without affecting the properties of Fibonacci cubes a new cube, known as extended Fibonacci cube, [7], has been introduced. Extended Fibonacci cubes are used to construct parallel machines with arbitrary size since it eliminates the restriction on the number of vertices.

A Fibonacci sequence is defined as $f_{0}=0 . f_{1}=1$,

$f_{i}=f_{i-1}+f_{i-2}$ for $i \geq 2$. The symbol $\|$ denotes concatenation operation for, $01 \|\{0,1\}=\{010,011\}$ and $01 \|\{\}=01$. The Fibonacci cube $\Gamma_{n}=\left(V_{n}, E_{n}\right)$ of order $n, n>1$, is defined recursively as $V_{n}=0\left\|V_{n-1} \cup 10\right\| V_{n-2}$, where $V_{n-1}$ and $V_{n-2}$ are the set of vertices of the order $n-1$ and $n-2$ respectively and there is an edge between two vertices if their binary representations differ exactly in one position. The initial conditions are $\Gamma_{2}=(\{\lambda\}, \emptyset)$ and $\Gamma_{2}=(\{0,1\},\{(0,1)\})$. A Fibonacci cube of order $n$ has $f_{n}$ vertices where $f_{n}$ denotes the $n^{\text {th }}$ Fibonacci number [5]. The Fibonacci cube $\Gamma_{n}$ contains two disjoint subgraphs that are isomorphic to $\Gamma_{n-1}$ and $\Gamma_{n-2}$, and there are exactly $f_{n-2}$ edges connecting the two subgraphs. The degree of a vertex in the Fibonacci cube $\Gamma_{n}$, lies between $\left\lfloor\frac{n-2}{3}\right\rfloor$ and $n-2$, for $n \geq 3$ [5]. Fibonacci cubes of orders 2, 3, 4 are depicted in Figure 1.

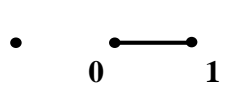

(a) (b)

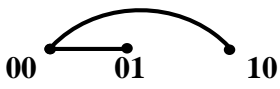

(c)
Figure 1: (a) $\Gamma_{2}$ (b) $\Gamma_{3}$ (c) $\Gamma_{4}$

Based on the Fibonacci sequence the extended Fibonacci cubes are defined by changing the initial conditions. 
A series of Extended Fibonacci Cubes, denoted by $E F C_{k}, k \geq 1$ is defined as $E F C_{k}(n)=\left\{V_{k}(n), E_{k}(n)\right\}, E F C_{k}(n-1)=$ $\left(V_{k}(n-1), E_{k}(n-1)\right)$, and $E F C_{k}(n-2)=\left(V_{k}(n-2), E_{k}(n-2)\right)$. Then $V_{k}(n)=0\left\|\mathrm{~V}_{k}(n-1) \cup 10\right\| V_{k}(n-2)$. Two vertices in $E F C_{k}(n)$ are connected by an edge in $E_{k}(n)$ if and only if their labels differ in exactly one bit. As initial conditions for recursion, $V_{k}(k+2)=\{0,1\}^{k}, V_{k}(k+3)=\{0,1\}^{k+1}$ where $\{0,1\}^{k}$ denotes the set of binary strings of length $k$ [7].

A Fibonacci cube $\Gamma_{n}$ is a proper subgraph of $E F C_{1}(n)$, for $n \geq 4$. For any $n \geq i+3$ and $n \geq j+3, E F C_{i}(n)$ is a proper subgraph of $E F C_{j}(n)$ if $i<j$. The degree of vertices in $\operatorname{EFC}_{k}(n)$ is between $\left\lceil\frac{n-(k-1)}{3}\right\rceil+(k-1)$ and $n-2$.

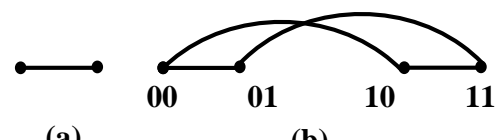

(a)

(b)

Figure 2: (a) $E F C_{1}(3)(b) E F C_{1}(4)$

The vertices of an $\operatorname{EFC}_{k}(n)$ are labelled with binary strings of length $n-2$, where the first $n-k-2$ bits represent a Fibonacci number and the last $k$ represents a binary number. The number of vertices in $\operatorname{EFC}_{k}(n)$ is $2^{k} f_{n-k}$, where $f_{n-k}$ is the $(n-k)^{\text {th }}$ Fibonacci number, $n>k+1$. The diameter of $\operatorname{EFC}_{k}(n)$ is $n-2$ for all $k \geq 0$ [8]. An extended Fibonacci cube $\operatorname{EFC}_{0}(n)$ is a Fibonacci cube $\Gamma_{n}$. $E F C_{1}(n)$ and $E F C_{2}(n)$ are shown in Figures 2 and 3 .

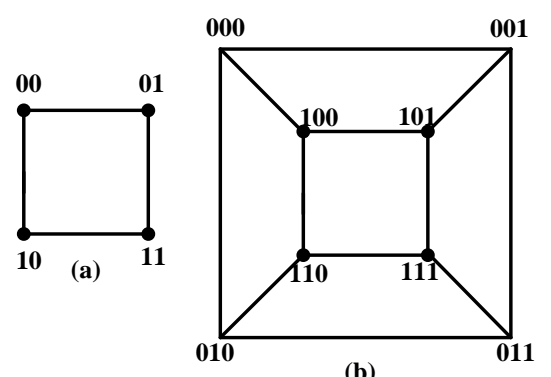

Figure 3: (a) $\mathrm{EFC}_{2}(3)$ (b) $\mathrm{EFC}_{2}(4)$

Theorem 2.1: Let $G=E F C_{2}(n)$. Then $\operatorname{ir}(G)=3$ for $n \geq 5$.

Proof: Vertices of $G$ are denoted by $a_{1}, a_{2}, a_{3}, \ldots, a_{m}$ where $m$ is the number of vertices in $G$ and any two vertices in $G$ are connected if and only if their Hamming distance is one. Consider $W=\left\{a_{1}\right\}$. Then there exist vertices $a_{i}, 2 \leq i \leq m$ and $i$ $\neq m-1$ with identical representation with respect to $W$. Thus $W$ does not resolve $G$ as an $i r$-set. Therefore, $\operatorname{ir}(G)>1$.

Include $a_{3}$ into $W$, that is, $W=\left\{a_{1}, a_{3}\right\}$. The vertices $a_{2 i}$, $1 \leq i \leq \frac{m}{2}$ are at equidistant from both $a_{1}$ and $a_{3}$. Hence $\operatorname{ir}(G)>$ 2 .

Now include $a_{m}$ in $W$. It follows that $d\left(a_{m-4}, a_{m}\right)=1$.

Then for $1 \leq i \leq \frac{m}{4}$,

$$
d\left(a_{4 i-2}, a_{m}\right)=(m / 4)-(i-1)+d\left(a_{m-4}, a_{m}\right)
$$

Similarly for $1 \leq i \leq\left(\frac{m}{4}\right)-2$,

$$
d\left(a_{4 i}, a_{m}\right)=(m / 4)-2-(i-1)+d\left(a_{m-4}, a_{m}\right)
$$

$d\left(a_{m-2}, a_{m}\right)=3+d\left(a_{m-4}, a_{m}\right)$

Thus any two vertices have distinct $W$-coordinates and $W$ resolves $G$.

Further the vertices $a_{1}, a_{3}$ and $a_{\mathrm{m}}$ are non-adjacent vertices in $G$. Therefore $W$ is a minimum independent set. Hence $W$ resolves $G$ as an independent resolving set and $\operatorname{ir}(G)=3$.

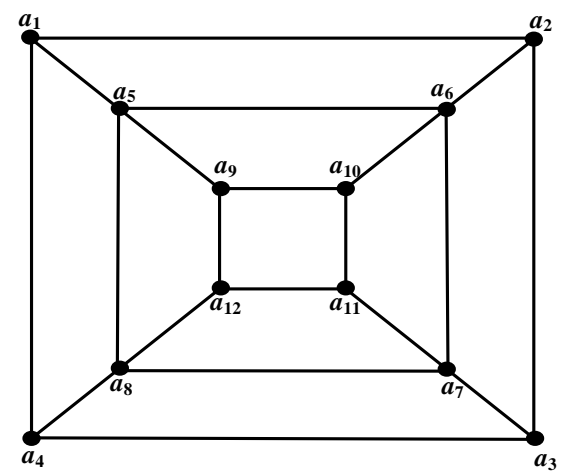

Figure 4: $E F C_{2}(6)$ with its resolving set $\left\{a_{1}, a_{3}, a_{12}\right\}$

Lemma 2.2: Let $G=E F C_{1}(n)$. Then $\operatorname{ir}(G) \geq n-3$ for $n \geq 5$.

Proof: Let $W=\left\{a_{k}\right\}$ where $k=\left\{\begin{array}{l}2 i+2,1 \leq i \leq\left\lfloor\frac{n}{2}\right\rfloor-1 \\ 4 i+4,1 \leq i \leq\left\lfloor\frac{n}{2}\right\rfloor-2\end{array}\right.$

be an independent set in $G$ such that $|W| \leq n-4$. Then there exist vertices $\left\{a_{2 n-9}, a_{m}\right\}$ which have identical representation with respect to $W$. Thus $W$ does not resolve $G$ and $\operatorname{ir}(G) \geq n-3$.

Theorem 2.2: For $G=E F C_{1}(n), \operatorname{ir}(G)=n-3$ for $n \geq 5$.

Proof: By Lemma 2.2, $\operatorname{ir}(G) \geq n-3$.

Let us assume that $\operatorname{ir}(G)=n-3$. We will prove this by induction on the number of vertices $n$.

Let $G=E F C_{1}(5)$ and $W=\left\{a_{4}, a_{6}\right\}$ be an independent resolving set in $G$. Then it can be easily verified that every vertex in $G$ is resolved by $W$. Thus $W$ is an ir-set and $\operatorname{ir}(G)=2$. Figure 5 represents the distinct representation in $W$ with respect to $G$. Now let us assume that the result is true for $\operatorname{EFC}_{1}(n)$.

$$
W=\left\{a_{k}, a_{j}\right\} \text { where } k=\left\{\begin{array}{l}
2 i+2,1 \leq i \leq\left\lfloor\frac{n}{2}\right\rfloor-1 \\
4 i+4,1 \leq i \leq\left\lfloor\frac{n}{2}\right\rfloor-2
\end{array}\right.
$$

and $j=m\left(E F C_{1}(n-1)\right)+2$ is an independent resolving set of $\mathrm{G}$ where $m\left(E F C_{1}(n)\right)$ denotes the number of vertices in $\operatorname{EFC}_{1}(n)$.

By the structure of extended Fibonacci cubes, $E F C_{1}(n+1)$, contains a copy of $E F C_{1}(n)$ and $E F C_{1}(n-1)$. Now we have to show that $W$ is an independent resolving set of $\operatorname{EFC}_{1}(n+1)$. That is, we have to prove that any two vertices, say, $x$ and $y$, do not have same distance with respect to $W$.

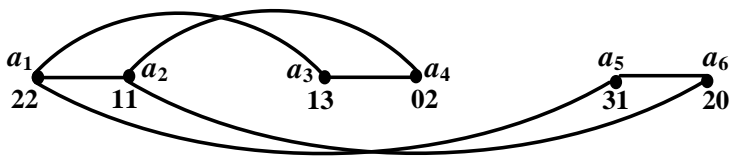

Figure 5: $E F C_{1}(5)$ and its distinct representations with respect to $W$ 
Case i: If $x, y \in E F C_{1}(n)$ (or) $x, y \in E F C_{1}(n-1)$, by induction hypothesis $W$ resolves $x$ and $y$.

Case ii: If $x \in E F C_{1}(n)$ and $y \in E F C_{1}(n-1)$.

In this case,

$$
\begin{aligned}
d\left(x, a_{j}\right) & =d\left(x, a_{2 p+j-1}\right)+d\left(a_{2 p+j-1}, a_{j-1}\right)+d\left(a_{j-1}, a_{j}\right) \\
& =d\left(x, a_{2 p+j-1}\right)+d\left(a_{2 p+j-1}, y\right)+d\left(y, a_{j}\right) \\
& \neq d\left(y, a_{j}\right), \text { where } p=\left\lfloor\frac{x}{2}\right\rfloor
\end{aligned}
$$

Thus representations of $x$ and $y$ with respect to $W$ are distinct. Thus $W$ is an ir-set and $\operatorname{ir}(G)=n-3$.

Theorem 2.3: The independent resolving number of $\Gamma_{n}$ is given by

$$
\operatorname{ir}\left(\Gamma_{n}\right)=\left\{\begin{array}{l}
\left\lfloor\frac{n}{2}\right\rfloor-1, \text { if } n \text { is even } \\
\left\lfloor\frac{n}{2}\right\rfloor, \quad \text { if } n \text { is odd }
\end{array}\right.
$$

The proof of this theorem is obtained by using the same strategy as in Theorem 2.2.

\section{CONCLUSION}

The exact values of independent resolving number for Fibonacci and extended Fibonacci cubes are determined. The problem is open for other networks like hypercube, hexagonal, etc.

\section{REFERENCES}

[1] Saenpholphat, V., Zhang, P., Conditional resolvability of graphs: a survey. IJMMS vol. 38, pp. 1997-2017, 2003.

[2] Z. Beerliova, F. Eberhard, T. Erlebach, A. Hall, M Hoffman and M. Mihalak, Network Discovery and Verification, IEEE Journal on Selected Areas in Communications, Vol.24, No. 12, pp. 2168-2181, 2006.

[3] Slater, P.J., Leaves of trees. Congr. Numer., 14, pp. 549$559,1975$.

[4] Y. Saad and M.H. Schultz, Topological Properties of Hypercubes, IEEE Trans. Computers, vol. 37, no. 7, pp. $867-872,1988$

[5] Hsu, W. J., Fibonacci cubes - a new interconnection topology, IEEE Trans. Parallel and Distributed Systems, 4, no.1, pp. 3-12, 1993.

[6] Hsu, W. J., Fibonacci cubes - Properties of an Asymmetric Interconnection Topology, Proc. Inter. Conf. on Parallel Processing, pp. 1249-1268, 1991.

[7] J.Wu., Extended Fibonacci Cubes, IEEE Trans. Parallel and Distributed Systems, vol.8, no.12, pp. 1203$1210,1997$.

[8] Ioana Zelina, A survey on cycles embeddings in Fibonacci and extended Fibonacci cubes, Creative Math. \& Inf., vol. 17 , no. 3 , pp. $548-554,2008$. 